\title{
Estimation of Magnetic Microwire Mechanical Properties by FEM Modeling
}

\author{
M. Spodniak*, K. Semrád, M. Šmelko, L. Fözö, R. Andoga, K. Draganová \\ AND S. SZABO \\ Faculty of Aeronautics, Technical University of Košice, Rampová 7, 04121 Košice, Slovakia
}

\begin{abstract}
The article is focused on the development of a methodology for the magnetic microwire finite element model creation, which have a bright prospect in terms of monitoring the mechanical stress inside the composite materials. The finite element model is based on a real geometry of a particular magnetic microwire. The glass coating of the magnetic microwire was mechanically removed and the information about the geometry was obtained by the microscope. Based on the photos the 3D model was created and the mesh of the magnetic microwire and the finite element model with boundary conditions were developed. The most important part of the article deals with the modelling of the magnetic microwire contact surfaces and the development of their finite element mesh. Once the finite element model is done, all properties for the static stress analysis are applied. In this context, the static stress analysis is performed and the results from the ANSYS APDL are presented. The article summarizes the new approach in investigation of magnetic microwire mechanical properties. The proposed finite element model can be also used for further investigation of magnetic microwires.
\end{abstract}

DOI: 10.12693/APhysPolA.137.674

PACS/topics: magnetic microwire, finite element method (FEM), mechanical stress

\section{Introduction}

Nowadays many different materials for the construction of modern aircraft are used. These materials can be classified into the four main categories such as metal, composite, ceramics, and plastics. Each of the mentioned material has its specific characteristics. The most suitable material for the aviation industry seems to be the composite because of its advantages and the possibility to design aircraft as light as possible. Composite materials have a combination of a number of advantageous properties for which they are used in the production of modern aircraft. However, they also have a number of disadvantages. An undesirable property of the composite materials is their inhomogeneity, which can lead to the sudden cracking in the material as a result of the exceeded maximum load. One of the possible ways to prevent these unfavourable properties is to monitor the mechanical stress inside the composite structure using magnetic microwires [1]. The usage of magnetic microwires in the role of a sensitive part of a mechanical stress sensor can ensure that the cracks between the particular layers can be revealed. Magnetic microwires can be implemented into the composite structure during the manufacturing process and because of their small dimensions they have only negligible influence on the structure of the composite materials [1-3].

*corresponding author; e-mail: miroslav.spodniak@tuke.sk

\section{The metal core and glass coating connection investigation}

Before performing the finite element method (FEM) calculations, it is necessary to know the exact geometry of the magnetic microwire. In our case the magnetic microwires consist of a metal core and a glass coating. The most important information for examining the stress is the connection of the core and the glass of the magnetic microwire. Therefore the glass of the magnetic microwire was mechanically removed. The metal core without glass coating was observed under the electron microscope as shown in Fig. 1, left [3].

\section{3D model of magnetic microwire}

Nine photos created from observation (Fig. 1, middle) were imported into the CAD program. At first, a $3 \mathrm{D}$ model of the microwire core was created without the contact surfaces. In Fig. 1, right the identical sketch is shown copying the obtained photo on the surface of
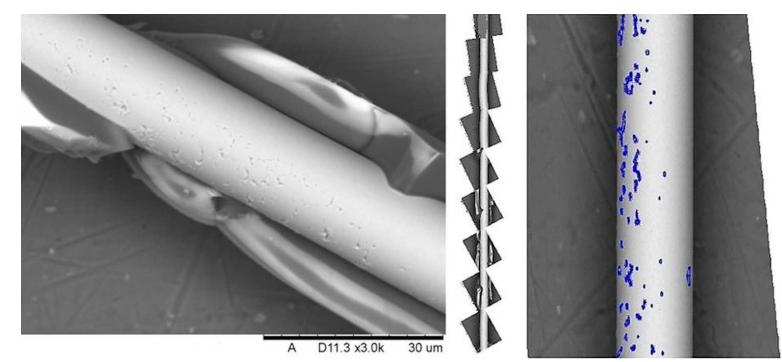

Fig. 1. Core and glass coating connection investigation. 


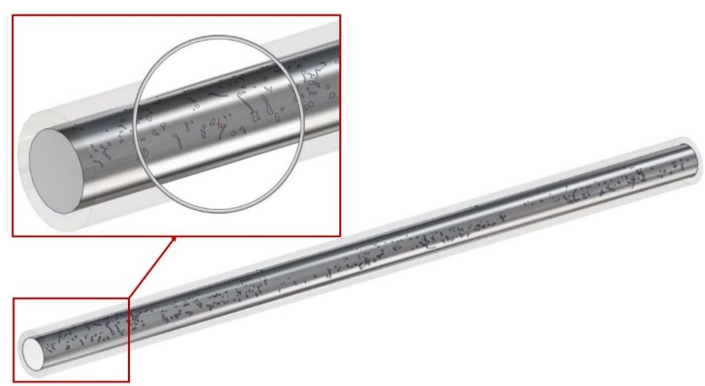

Fig. 2. Magnetic microwire 3D model.

the 3D model of the magnetic microwire core. In an analogous way 900 small surfaces on the $3 \mathrm{D}$ model for each photo were created. The cylinder core is a cut with the final sketch, which represents a planar connection between the core and the magnetic microwire glass coating [4]. When it comes to 3D model enlargement the model is magnified by 1000 in comparison with real magnetic microwire.

The length of the model in reality in $5 \mathrm{~mm}$ and the diameter is $0.035 \mathrm{~mm}$ but after enlargement it is $5000 \times 35 \mathrm{~mm}$. The glass coating, as well as the core were created in the CAD program, which is only a hollow cylinder. The complete 3D geometry of the microwire, magnified 1000 times, is shown in Fig. 2.

Based on the 3D model, it is possible to perform the FEM analysis and to examine the transfer of the mechanical stress from the composite through the glass to the core of the magnetic microwire as in the real conditions. It also will be possible to show if these small contact regions withstand the high loads [4].

\section{Meshing of magnetic microwire FEM model}

Based on the geometry of the 3D model, it is possible to create a FEM model that consists of the hexa and tetra elements, which is a hybrid generation of the finite element model. The elements in the area of the interest consist of the hexa elements and the core is meshed with the tetra elements. The geometry is divided into nine segments as the photos from the microscope were taken. Each segment is meshed on the surface of the core. Then the 2D surface mesh of the entire core is created (Fig. 3). The $2 \mathrm{D}$ mesh is mapped into two layers of the $3 \mathrm{D}$ mesh elements for the magnetic microwire core [5].

The next step is to create a part of the tetra mesh. From the point of view of meshing the time is considerably saved and the quality of the model is not reduced by the tetra elements as they are far from the area of interest. The magnetic microwire core is completely modeled and it is necessary to mesh second part of the magnetic microwire, which is the glass coating. The glass coating was meshed analogically to the core. The $2 \mathrm{D}$ elements were mapped into five rows of the $3 \mathrm{D}$ hexa elements with the SOLID45 properties [6]. Once the glass and the core are meshed, it is possible to model the contacts between them. In order to model contacts it is necessary

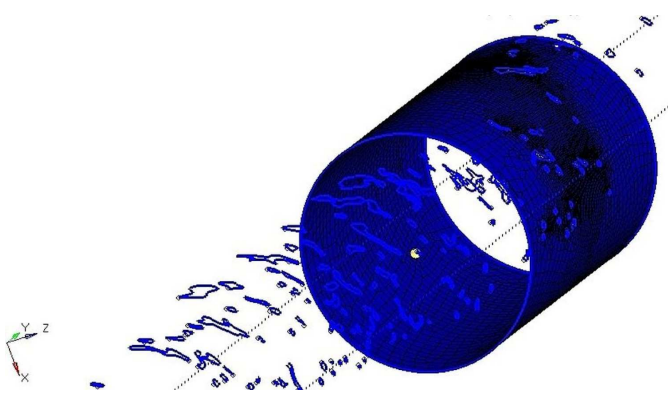

Fig. 3. 2D mesh of magnetic microwire.

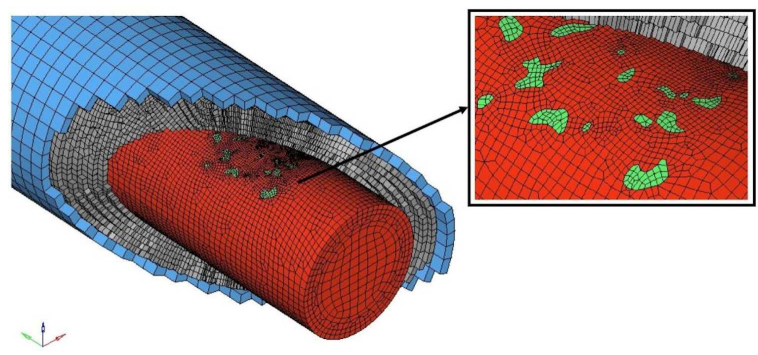

Fig. 4. Finite element model of magnetic microwire.

to mark each element of the mesh in the contact surface regions between the core and the coating. Consequently, the contacts with the corresponding properties are modeled. There are over 900 small contact surfaces between the glass and the metal core, which means thousands of finite elements. It is necessary to mark every single element for contact modeling, which means a large amount of work and time. The final step in the meshing of the core and coating of the magnetic microwire is to mesh a thin composite layer. It will ensure that the boundary conditions can be applied. The final FEM model with the partial section is shown in Fig. 4.

Mesh in Fig. 4 with the red color is the core, the grey part is the glass coating, blue color was used for the composite material, and the green color shows the contact between the core and the coating of the magnetic microwire. The microwire glass is bonded to the core of the microwire by the contacts and all nodes of the core and of the glass are identical on the surface in order to create the contact pairs.

In order to decrease the solving time it is possible to create only a half FEM model of the magnetic microwire since the model is symmetric (Fig. 5). Solving a rotationally symmetric task, it is necessary to define the cyclic symmetry appropriately using the commands in the ANSYS APDL program that are part of the calculation script. Under these conditions the microwire is behaving like in real conditions even though it is simplified.

\section{Boundary conditions and solving}

The ANSYS APDL program was selected for solving. The purpose of the calculation is to observe the transfer of the mechanical stress form the composite to the core 


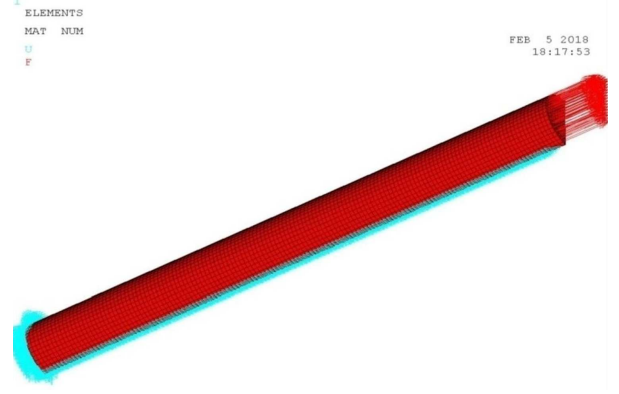

Fig. 5. Boundary conditions on magnetic microwire.

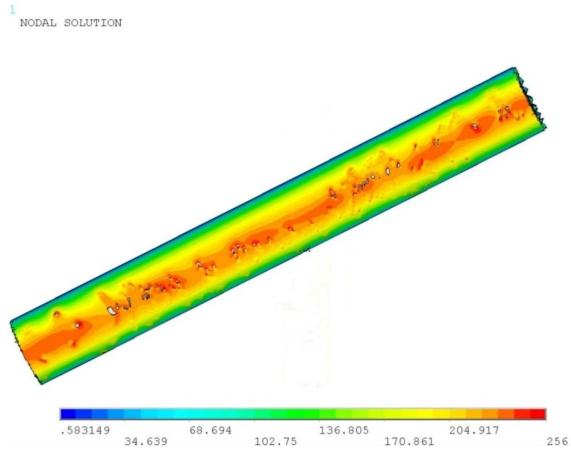

Fig. 6. Von Mises stress on the surface of magnetic microwire [MPa].

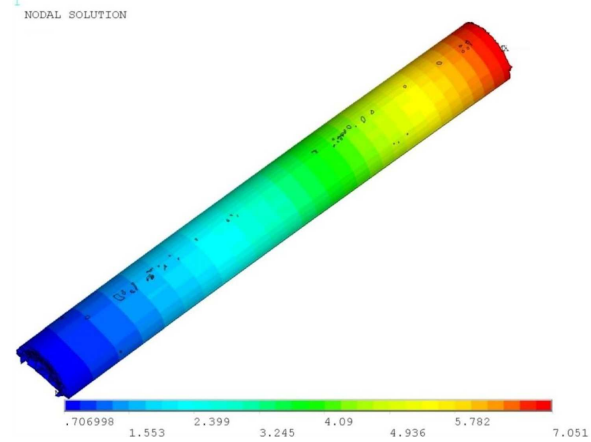

Fig. 7. Deformation of magnetic microwire [mm].

of the magnetic microwire. The element type for the core, glass, and composite layer is SOLID45, which is intended for the 3D solid structural models. For the contacts the elements CONTA173 and TARGE170 were selected [6]. Consequently, it is possible to define boundary conditions. One end is fixed and at the other end the force with magnitude of $100 \mathrm{kN}$ is applied using the created node sets (Fig. 5).

The results are presented in Fig. 6, which shows the resulting Von Mises stresses on the magnetic microwire core surface. In Fig. 6, there is the section of the central part of the microwire without the glass coating and the composite structure in order to show the stress on the core. Since the applied force is in $[\mathrm{N}]$ and the dimensions are in $[\mathrm{mm}]$, the resulting stress is in $[\mathrm{MPa}]$.
The maximal stress reaches the value of $256 \mathrm{MPa}$ in the small surface regions that connect the core and coating of the microwire. The high stress concentration can be seen in the small surface contact regions. This high stress concentration may in extreme cases lead to the breaking of the contacts between the core and the glass of the magnetic microwire $[5,6]$. The information about the direction of the deformation and the correctness of the applied force is also clearly seen in Fig. 7. The red colour shows the maximum deformation values of the section.

\section{Conclusion}

The main aim of the proposed article is to present a closer view of the development of the finite element model of the magnetic microwire. The model based on the geometry of the real magnetic microwire and the long process of meshing is briefly described in the article. Result of the presented work is the valuable model that can be used for the scientific investigation of the magnetic microwire properties.

The method used to estimate the maximal stress and deformation of the magnetic microwire discussed in this paper can be used in other applications of the FEM methodology in order to predict the mechanical or magnetic properties. Although there are boundary conditions that should be changed for other application, the FEM model is developed and can be used for other analysis. Based on the developed model the FEM analysis can be carried out in order to investigate not only the mechanical stresses but also other physical or magnetic properties in industrial or biomedical applications, thermal analysis, etc.

\section{Acknowledgments}

This work was supported by the APVV-17-0184, VEGA No. 1/0374/17, KEGA No. 052TUKE-4/2018, and KEGA No. 058TUKE-4/2018 projects.

\section{References}

[1] M. Šmelko, M. Spodniak, K. Semrád, P. Tulipán, P. Lipovský, V. Moucha, in: 2018 XIII Int. Sci. Conf. - New Trends in Aviation Development (NTAD), Košice (Slovakia), 2018.

[2] I. Baraban, L. Panina, A. Litvinova, V. Rodionova, J. Magn. Magn. Mater. 481, 50 (2019).

[3] M. Šmelko, D. Praslička, K. Draganová, P. Lipovský, V. Kán, J. Bajús, UPB Sci. Bull. Series D Mech. Eng. 78, 1 (2016).

[4] K. Semrád, P. Lipovský, J. Čerňan, M. Jurčovič, Proced. Eng. 96, 428 (2014).

[5] K. Semrád, K. Draganová, Metalurgija 56, 1 (2017).

[6] S. Moaveni, Finite Element Analysis Theory and Application with ANSYS, Prentice-Hall, New Jersey 1999. 